\title{
Noch einmal der Tod der Söhne Zebedaei.
}

Von E. Schwartz in Freiburg i. B.

F. Spitta hat im 1. Heft des 11. Jahrgangs dieser Zeitschrift behauptet, ich habe ohne zureichenden Grund, durch Wellhausens Deutung von Mk 10, $35 \mathrm{ff}$. verführt, die Hypothese aufgestellt (Abhandlgn. d. Gött. Ges. d. Wiss. N. F. VII, 5; Nachr. d. Gött. Ges. d. Wiss. 1907, 266 ff.), daß die Söhne Zebedaei im Anfang des Jahres 44, kurz nach der Zusammenkunft des Paulus mit den "Säulen" in Jerusalem, auf Befehl Agrippas I. hingerichtet seien und alles, was von dem Aufenthalt des greisen Johannes in Ephesus erzählt wrerde, ausnahmslos in das Reich der Legende verwiesen werden müsse. Ich bin kein Freund von Erwiderungen und Spittas Argumente würden an und für sich mich um so weniger veranlaßt haben, noch einmal auf eine Sache zurückzukommen, die ich im wesentlichen für entschieden halte, als ich den beweglichen Worten meines ehemaligen Kollegen, dessen Freundschaft ich stets hochgeschätzt habe, nur das offene Bekenntnis entgegensetzen kann, daß meine Art, mit der Überlieferung und den Texten umzugehen, von der seinen $z u$ verschieden ist, als $\mathrm{da} B$ wir hoffen könnten, einander $z u$ überzeugen. Andrerseits halte ich die Legende von dem ephesischen Johannes und die damit .zusammenhängende, wie es scheint unausrottbare Neigung, von dem apostolischen Ursprung des vierten Evangeliums irgendwelche, auch noch so wertlose Fetzen zu retten, für ein so schweres Hindernis der kirchengeschichtlichẹn Forschung, daß für die echte und wahre Überlieferung nicht oft und scharf genug gekämpft werden kann, und da ich meine Anschauungen in einigen Nebenpunkten stützen und ergänzen kann, so ergreife ich noch einmal das Wort. Um mich möglichst wenig wiederholen zu müssen, setze ich die Kenntnis meiner obenerwähnten Aufsätze bei meinen Lesern voraus.

Die Überlieferung, daß Johannes und Jakobus, die Söhne Zebedaei, als Märtyrer gestorben sind, ruht auf dem sicheren, unanfechtbaren 
Zeugnis des Papias und des sog. syrischen Martyrologiums, d. h., wie ich schon öfters bemerkt habe, des vortheodosianischen Festkalenders von Konstantinopel; dazu kommt noch Aphraates, der, wie Wellhausen mir mitteilt, an einer Stelle $\left(417^{10}\right)$ bemerkt, daß es außer Stephanus, Petrus und Paulus nur zwei apostolische Märtyrer gebe, Jakobus und Johannes. Es heißt die Sachlage arg verkennen, wenn Spitta diese Tradition mit der Bemerkung zu diskreditieren sucht, daß sic von Schriftstellern vertreten werde, „die unter allen Umständen jünger seien als der Verfasser der Apostelgeschichte“. Einmal zugegeben, daß Papias, dessen Zeit ich gestehe nicht genau angeben $z u$ können, wirklich jünger ist als die letzte Redaktion der Apostelgeschichte, die ich ebenfalls nicht chronologisch fixieren kann, so kommt darauf für diese Frage nichts an: denn mit dem Alter des Papias ist über das der Tradition, die er weitergibt, außer dem terminus ad quem nichts entschieden, und kein Mensch kann die These ernsthaft verfechten, daß alle durch Papias gesammelten und erhaltenen Traditionen jünger sein müßten als die abschließende Bearbeitung der Apostelakten. Wenn ferner die Gemeinde von Aelia und ihr folgend andere den Märtyrertod der beiden Zebedaeussöhne noch im vierten Jahrhundert feierten, so begreift man schwer, wie ein solcher Brauch in späterer Zeit aufkommen konnte, wo die kanonischen Apostelakten und die Legende vom ephesischen Johannes alle Gemüter beherrschten, dagegen ist es nach allen Gesetzen historischer Kritik leicht verständlich, daß eine uralte, bei der Gründung von Aelia noch lebendige Tradition sich im Schutz des Kultus gewissermaßen versteinert erhielt, nachdem sie in der Literatur von Neubildungen überwuchert war. Noch im dritten Jahrhundert knüpften die Erzählungen, die in Smyrna über die Anfänge der dortigen Gemeinde umliefen, nicht an Johannes, sondern an Paulus an, ${ }^{1}$ und im zweiten, zu einer Zeit, wo, wenn die Legende recht hätte, Johannes vor wenigen Jahren ein langes Leben in Ephesus beschlossen haben mußte, gedenkt Ignatius in seinem langen Briefe an die Epheser dieser glorreichsten Erinnerung mit keinem Worte und redet

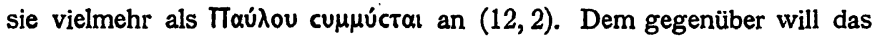
unbestimmte Gerede des Irenaeus von dem „Apostelschüler“ Polykarp wenig besagen (Eus. KG 4, $\left.14^{3}\right)$; hat ihm doch schon Euseb $\left(3,39^{1} \mathrm{ff}\right.$.) nachgewiesen, daß er Papias mit Unrecht für einen Schüler des Johannes ausgibt. Ich will ihm noch gar nicht einmal das Mißtrauen entgegenbringen, das er als Ketzerbeștreiter und dogmatischer Verfechter der apostolischen Sukzession der Bischöfe reichlich verdient, und der Be-

1 Vgl. Corssen in dieser Zeitschrift 5, 299. 
hauptung den Glauben nicht versagen, die er in einem polemischen Brief (Eus. KG 5, $20^{5}$ ff.) aufstellt, daß er als Knabe Polykarp persönlich gehört habe; daß dieser wirklich von seinem Verkehr mit dem Apostel Johannes erzählt hat, wird durch das Schweigen des Ignatius in seinen Briefen an ihn und die Smyrnäer und des Pionius in seiner Vita bündig widerlegt: der glaubenseifrige Bischof hat das unsichere, verblassende $\mathrm{Er}$ innerungsbild mit eigenen Erfindungen aufgefrischt, wie es $z u$ allen Zeiten vorkommt.

Ständen sich nur die Überlieferung von dem Märtyrertode der Zebedaeussöhne und die Legende von dem ephesischen Aufenthalt des Johannes gegenüber, so wären die Zwveifel an jener allenfalls verständlich; sie sind es nicht mehr, wenn das ausdrückliche Zeugnis des Markusevangeliums (10, $35 \mathrm{ff}$.) jener Überlieferung zu Hilfe kommt. Hatte Jesus zweien seiner ersten Jünger mit Unrecht geweissagt, daß sie den Tod von Henkershand erleiden würden wie er, dann weiß ich nicht, wie eine solche falsche Weissagung ins Evangelium gelangen konnte: ich kann mich nicht ohne Grund rühmen, den Worten des Evangeliums nicht so leichthin den Glauben zu versagen, wie es meine Gegner tun. Es ist eine leere Ausflucht, das Orakel um der Apostelakten willen nur auf Jakobus $\mathrm{zu}$ beziehen: warum ist denn Johannes mit genannt, warum die Geschichte zugespitzt auf das Sitzen zur Rechten und zur Linken des Messias? Die Doppelung des Lohnes setzt doch ein doppeltes Martyrium voraus. Vollends zu verurteilen - ich kann's nicht milder ausdrücken - ist eine Exegese, die den Kelch, den die Zebedaeussöhne trinken, und die Taufe, mit der sie getauft werden sollen, nicht vom Tode, sondern vom "Leiden" verstehen will. Dann darf natürlich auch Jesus nicht von seinem Tode, sondern nur ganz unbestimmt von einem Leiden während seines Lebens reden, als wenn die Kirche je unter dem Leiden Jesu etwas anderes als sein Sterben verstanden hätte und hätte verstehen können! Was aber an der Stelle Jesu recht ist, ist den Zebedaeussöhnen billig. Mit „Dulden“, mit „Selbstverleugnung“ und ähnlichen Tugenden einer gebildeten, bürgerlichen Ethik erwarb man sich in den kraftvollen Zeiten der Urgemeinde nicht den Anspruch auf die höchste Ehre, die es geben konnte, auf die Plätze zur Rechten und Linken des Herrn; dazu gehörte mehr, und.wenn die alten Christen das Gebet nicht ernst genommen hätten, sich selbst oder ihr Leben oder ihre Seele - für den Aramäer ist das ja alles dasselbe - dahinzugeben, so würden sie die Welt nicht aus den Fugen gebracht haben. Spitta hat seiner Sache keinen Dienst damit geleistet, daß er sich auf das alttestamentliche, vermutlich von Jeremias (25) erfundene Bild beruft 
von dem "Taumelbecher", den Jahveh den Völkern reicht zur Strafe und zum Verderben ${ }^{\prime}$ : ich sehe nicht, wie damit bewiesen werden soll, daß an der Markusstelle der Kelch, den Jesus trinkt und die Zebedaeussöhne ihm nachtrinken, „Leid" bedeutet und nicht "Tod"; dem Untergang von Städten, Völkern, Reichen entspricht der Tod des Individuums. Setzt man „Leid“, um die modernen, sentimentalen Nebenvorstellungen

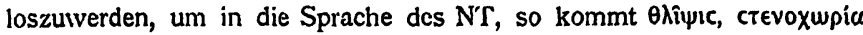
oder etwas derartiges heraus; mit gutem Recht konnte die Urgemeinde glauben, daß der Herr ihr derartiges geweissagt habe, aber die Versuchung lag ihr, wie die synoptische Überlieferung zeigt, fern, ihn selbst $z u$ den $\theta \lambda_{1} \beta o ́ \mu \in v o t ~ z u$ rechnen. Ein Leben voller Leid und Qual hatte er nicht geführt; sein Leiden war sein Tod: der war der Kelch, den $z \mathrm{u}$ trinken er sich fürchtete, wie er selbst sagt (Mk 14,36). Polykarp dankt in seinem letzten Gebet Gott dafür, daß er ihm in der Schar der Märtyrer Anteil an dem Kelche Christi gegeben hat ${ }^{2}$ : da hat das von Jesus selbst neu geprägte Bild des Todeskelches von seinem Vollgehalt noch nichts verloren. Wenn man also in dem Orakel über die Zebedaeussöhne, das, wie alle zugeben, in die Urzeit gehört, das Bild seines ihm aufgeprägten Wertes beraubt und zur.abgeschliffenen Scheidemünze devaluiert, so ist das ein Verstoß gegen die elementaren Gesetze der Auslegung, gegen den ich nicht aufhören werde zu protestieren. Wie unter dem Kelch der Todeskelch, so ist unter der Taufe natülich die Bluttaufe $z u$ verstehen. Die Taufe auf den Tod Jesu ist später durch Paulus (Rom 6, 2) ein beliebter Ausdruck der christlichen Mysteriensprache geworden; auch von dieser Formulierung abgesehen, stimmt die Taufe auf den Namen Jesu nicht recht zu dem Vergleich von Jesu eigenem Tod mit einer Taufe. In dem Orakel ist eben das Martyrium der Jünger die Hauptsache, der Tod Jesu das was damit verglichen wird; daher wird die Bluttaufe, die streng genommen nur auf die Jünger paßt, auf den Herrn mit übertragen. Matthaeus ist das so anstößig getvesen, daß er $(20,22 \mathrm{ff}$.) nur den Todeskelch stehen läßt, aber die Taufe streicht.

Spitta macht ein großes Weisen davon, daß Jesus von seiner Passion im Praesens rede, also seinen erst bevorstehenden Tod und darum auch den der Zebedaeussöhne nicht meinen könne (Mk 10,38): Júvac $\theta \epsilon$ mê̂v tò

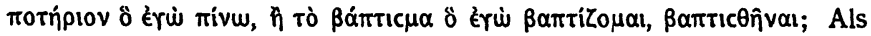

1 Die Stellen bei Olshausen zu Ps 60, 5 und Smend zu Ez 23, 31.

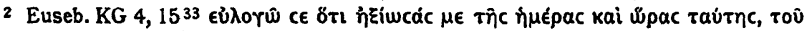

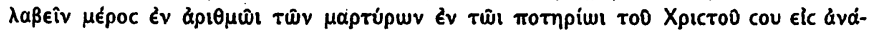

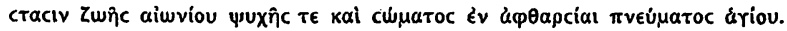


w'enn das griechische Praesens hier nicht der Ersatz für die aramäische Partizipialkonstruktion wäre, die an und für sich keine bestimmte Zeit bedeutet, sondern ihren temporalen Wert durch den Zusammenhang erhält. Mit noch weniger Recht will er durch Berufung auf das Praesens Ex $x$ in der Stelle Lk 12,50 die Deutung der Taufe auf den Tod Jesu

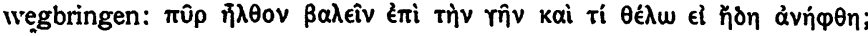

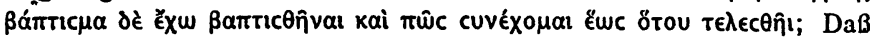
Éxw mit dem Infinitiv Aoristi futurischen Sinn hat, eine Sprachbildung vergleichbar dem Futur der romanischen Sprachen, läßt sich so wenig hinwegräsonnieren wie daß der Satz mit éwc nur bei einer Aussicht in die Zukunft Sinn erhält. Freilich reimen sich der stolze Hinweis auf die Vorzeichen der Parusie und die Angst vor dem eigenen Ende nicht mit einander: es läßt sich nun einmal nicht ändern, daß Lukas hier ungleichartige Trümmer zusammengekittet hat, am wenigsten durch solche Interpretationskünste wie sie Spitta vorschlägt, daß die Taufe hier das Wasser bedeuten soll, unter das die Gegner Jesu Werk zu setzen sich bemühen. Die Belegstellen, die er zitiert, Sir 3, 30. Cantic 8, 6f., sehen ja gelehrt aus, aber was geht der Vergleich des die Sünde tilgenden Almosens mit dem die Flamme löschenden Wasser oder die Ströme, die das Liebesfeuer nicht fortspülen, die Taufe an, die Jesu bevorsteht, und seit wann sind Taufbad und Wassersflut dasselbe Bild? Trägt man Spittas Auslegung vón Lk 12,50 in das Orakel von den Zebedaeussöhnen hinein - und diese Konsequenz muß er sich gefallen lassen, wenn der exegetische Exkurs nicht zwecklos sein soll - so kommt heraus, daß Jesus von ihnen verlangt, sie müßten sich's gefallen lassen, daß ihr Werk von den Gegnern ebenso unter Wasser gesetzt werde wie sein eigenes. $\mathrm{Da}$ hat die alte Kirche das Orakel unendlich viel besser verstanden, wenn sie es mit der ephesischen Tradition in der Weise ausglich, daß sie erfand, Johannes habe ohne Schaden den Giftbecher getrunken oder ein Bad in siedendem Ö $1^{1}$ ausgehalten; so ist doch der Grundgedanke, daß Jesus von einem Martyrium redet, scharf und präzise festgehalten. Übrigens gesteht Spitta selbst $z u$, daß Matthaeus unter dem Becher den Todesbecher verstanden hat, weil er für die oben aus-

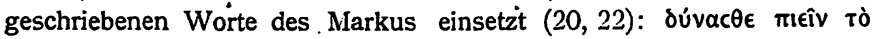

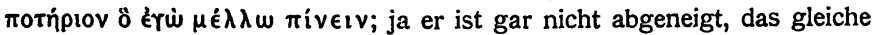
auch für Markus einzuräumen, und macht nicht mit Unrecht darauf aufmerksam, daß das Sterben Jesu der Leitgedanke ist, der das Orakel über die Zebedaeussöhne nit der vorhergehenden, sehr detaillierten Weis-

1 Die Oltaufe kennt schon Tertullian (de praescr. 36). 
sagung von Jesu eigener Passion $(10,32-34)$ zusammenhält. Wenn aber die Evangelisten unter dem Becher, den Jesus trinkt, seinen Tod verstanden haben, so gilt die gleiche Deutung auch fir den Becher, den die Zebedaeussöhne trinken sollen: sie haben also ihr Martyrium entweder für wahr gehalten, und dann wiegt ihr Zeugnis sehr schwer, oder es erfunden; mit den Konsequenzen, die sich daraus für die evangelische Überlieferung ergeben, mögen meine Gegner sich auseinandersetzen. Die Ausflucht, die einem Gnostiker Ehre machen würde, daß sie ein anders gemeintes Wort Jesu mißverstanden haben, führt aus dem Dilemma nicht heraus: es macht wenig Unterschied, ob man die Weissagung einer ganz bestimmten Tatsache, die sie Jesu in den Mund legen, auf Fiktion oder auf Mißverständnis zurückführt. Ganz davon zu schweigen, daß der gesamte Text in sich so klar, verständlich und geschlossen ist, daß nicht der geringste $Z$ wang vorliegt, an ihm herum zu experimentieren. Freilich nehmen die Zebedaeussöhne hier Jesu Weissagungen seines eigenen Endes als etwas Selbstverständliches ruhig hin, während die Jünger diese sonst nicht begreifen oder darüber entsetzt sind. Das liegt daran, daß die Weissagungen verschieden orientiert sind. Wenn Jesus nur von sich spricht, sollen sie den Einwand, daß er seinen Gegnern unterlegen sei und daher nicht der Messias gewesen sein könne, dadurch entkräften, daß diese Niederlage als vorher gewußt und gewollt hingestellt wird. Die Jünger begreifen das freilich nicht, denn ihr Glaube beginnt erst mit der Auferstehung. In dem Orakel über die Zebedaeussöhne geht das Interesse nach einer anderen Seite. Der Märtyrertod des Brüderpaares, das dem Herrn im Leben so nahe gestanden hatte, hat die Urgemeinde gewaltig aufgeregt, und es ist sicherlich ernsthaft darüber gestritten, ob sie den Ehrenplatz neben dem Herrn dadurch erworben hätten: die Geschichte will diese Debatten abschneiden aus dem sehr gesunden Gefühl heraus, daß der Märtyrerkult seine Gefahren hat und der Anspruch auf himmlische Ehrenplätze hienieden auch durch den Tod nicht erworben wird. In diesem Zusammenhang ist das Leiden und Sterben des Herrn etwas Gegebenes, über das nicht reflektiert werden kann, ohne die Pointe der Erzählung zu zerstören. Auch die Ermahnungen Jesu zur Nachfolge und zum Kreuztragen, die denselben Sinn haben wie die Worte, die er an die Zebedaeussöhne richtet, werden von den Jüngern sofort begriffen: sie sind ja aus dem Geist geboren, der nach Jesu Tode bei ihnen war. Man höre doch auf, diese schroffen und herben Ermahnungen zu trivialisieren, hinter den Todesweissagungen, die ihren Sinn verlieren, wenn sie nicht wunderbare Prophezeiungen sind, irgendwelche Banalitäten 
zu suchen, die der sog. „historische“ Jesus gesprochen haben könne, Dinge zu erfinden, an die die Evangelisten nie gedacht haben, und dem echten und tiefen Leben der Gemeinde, das in den Evangelien flutet, die Tür zu sperren: damit wird dem Glauben nicht geholfen und die Wissenschaft nicht aufgehalten.

Man würde nie Bedenken getragen haben, das Orakel über den Tod der Zebedaeussöhne so zu verstehen wie es gemeint ist, vielmehr die Überlieferung von ihrem Martyrium immer wieder als eine Bestätigung der evangelischen Überlieferung freudig begrüßen, wenn nicht das Evangelium Johannis wäre. Denen, die an'seinem apostolischen Ursprung festhalten, sollte man eigentlich die Schwungkraft des Glaubens zutrauen, daß sie es vor 44 verfaßt sein lassen: so mutig ist aber niemand mehr. Es wagt auch niemand, wenigstens niemand der wissenschaftlich ernst genommen werden will, das vierte Evangelium, so wie es ist, für die authentische Darstellung von Jesu Taten und Reden auszugeben, gegen welche die Synoptiker nicht aufkommen können, sondern man drückt sich um das böse Entweder - Oder herum mit Ausflüchten, die alle nicht besser sind als die alte von Irenaeus und Clemens aufgebrachte Hypothese, daß Johannes sein Evangelium zur Ergänzung der Synoptiker geschrieben habe, eine Hypothese, die schon daran scheitert, daß das vierte Evangelium so gut wie jedes andere Evangelium autonom sein und alle anderen überflüssig machen will. ${ }^{1}$ Auch der Versuch, den zuletzt Spitta unternommen hat, ist nicht neu, einen Kern herauszuschälen, der dann für echt ausgegeben werden kann. Dabei kann einzelnes richtig beobachtet werden; der apologetische Zweck aber hindert von vornherein, das Problem scharf zu fassen, und tatsächlich spricht das Wenige, was sich über die "Grundschrift" ausmachen läßt, klärlich dafür, daß dem Verfasser die Überlieferung gänzlich gleichgültig gewesen ist. Mag dem aber sein wie ihm wolle, wenn die Unmöglichkeit, das vierte Evangelium in vollem Umfang dem Zebedaeussohne Johannes zuzuschreiben, allgemein zugestanden wird, warum sträubt man sich gegen das richtige Verständnis des evangelischen Orakels, welches das Problem des vierten Evangeliums wenigstens von dem Phantom des apostolischen Ursprungs und damit von einer Fülle komplizierter Hypothesen befreit, warum fährt man fort um einer apologetischen Absicht willen, die völlig zu verwirklichen. man sich doch scheut, einen sonnenklaren Text mit schiefen und gezwungenen Interpretationen $\mathrm{zu}$ mißhandeln? Wie die ephesische Legende

1 Nachr. d. Gött. Ges. d. Wiss. 1908, 124 ff. 
entstanden ist, läbt sich nicht mehr vollständig aufklären, sie ist aber darum noch lange kcin solches Rätsel, wic es das evangelische Orakel und die von Papias und den Festkalendern aufbewahrte Tradition sein würde, wenn jene Legende Geschichte wäre. Zwei Momente sind auscinander zu halten, die Übertragung des Johannes nach Ephesus und die Umwandlung des Apostels in einen Schriftsteller. Jene ordnet sich sofort zusammen mit einem ähnlichen Prozeß, der in dem phrygischen Hierapolis vor sich gegangen ist; wie in Ephesus der Apostel Johannes, so wurde dort der Apostel und Evangelist Philippus mit seinen weissagenden Töcltern, dic im palaestinischen Caesarea gelebt hatten, annektiert. Darin lebt das Heidentum fort; Ephesus und Hierapolis sind alte Kultstätten und an Stelle der heidnischen Götter, die einst Segen und Verdienst gebracht hatten, sollten die Apostel als christliche Schutzpatrone treten: wie beim antiken Heroenkult, so ist an beiden Orten die Ubertragung vom Grabe ausgegangen. Die aus ihr hervorgewachsenen Legenden ivürden neben der älteren und richtigen Überlieferung ein harmloses Winkeldasein geführt haben, wenn der Name des Apostels Johannes nicht in eine merkwürdige literarische Bewegung hineingezogen und zur Etikette einer ganzen Reihe von Schriften gemacht wäre. Die Apokalypse und der erste johanneische Brief sind durch Interpolationen am Anfang zu Werken desselben Verfassers gestempelt, wie das Logosevangelium, dem der apostolische Ursprung vindiziert wird. Denn es hat einen Schluß erhalten, der es dem im Evangelium vorkommenden Lieblingsjünger zuschreibt und diesen zugleich als einen Jünger Jesu charakterisiert, der, anders als Petrus, kein Martyrium erlitten hat.

Ich habe früher über das Schlußkapitel nicht ganz richtig geurteilt, da ich es für einheitlich hielt; jetzt hat Wellhausen nachgewiesen (Ev. Johannes $98 \mathrm{ff}$.), daß verschiedene Hände an ihm tätig gewesen sind. Der zweite Teil des Kapitels, der mit dem ersten innerlich nicht zusammenhängt, enthält zunächst eine Doublette $(21,15-17)$ zu Mt 16, 17 ff.: Petrus wird, historisch richtig, als der Stifter der Urgemeinde hingestellt. Daran schließt sich die Weissagung seines Martyriums

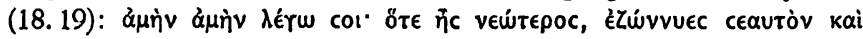

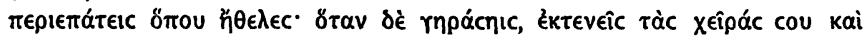

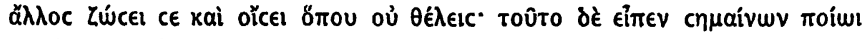

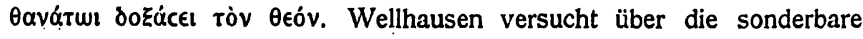

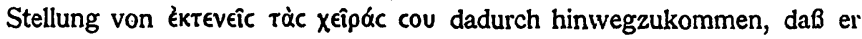
die Worte nicht auf die am Kreuz ausgereckten Arme bezieht, sondern auf die Hände, die der Delinquent vor der Hinrichtung zum Binden 
hinstreckt. Mich überzeugt das nicht; das ganze Kolon stört vielmehr den scharf ausgearbeiteten Gegensatz. Schief ist ferner, wie Wellhausen

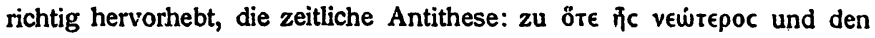
darauf folgenden Imperfekten paßt als Gegensatz nur vôv, oder es müßte umgekehrt dem ơtav mpácnıc im ersten Glied ein vôv mit dem Präesens entsprechen. Ferner kann die Bewegungsfreiheit, wenn sie dem gewaltsamen Tod entgegengestellt werden soll, nicht im Tempus der Vergangenheit stehen: ist Petrus als Hüter der Schafe Jesu nicht jahrelang "hingegangen wohin er wollte"? Mit diesen Worten hat also ein Bearbeiter sein Wesen getrieben; er wird die úvílaren und schiefen Hinweise auf das Martyrium des Petrus erst hineingebracht haben; sie stimmen ja auch zu der Aufforderung Jesu, die dann folgt (19), nicht: er kann Petrus doch nicht erst das Martyrium weissagen und dann, abrupt, ohne Anknüpfung an das Vorhergehende, es befehlen. Oder

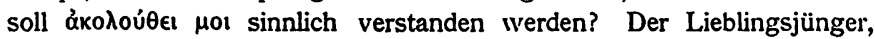
der nunmehr eingeführt wird, geht ja wirklich hinter Jesus her (20). Und doch zwingt der Gegensatz in 22 dazu, den Befehl "folge mir

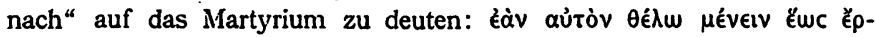

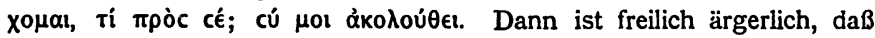
derselbe Jünger, der bildlich nicht nachfolgt, es sinnlich tut. Wie oben das Martyrium Petri, so ist hier das Gegenstück dazu, das "Bleiben“ des Lieblingsjüngers, einem Zusammenhang aufgepfropft, in dem es nicht gewachsen ist. Diese Beobachtung hilft vielleicht dazu, das Rätsel, das über den Versen 22 und 23 liegt, seiner Lösung näher zu bringen.

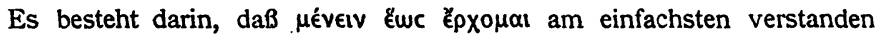
wird von der Fortdauer des Lebens bis zur Parusie, dann aber die Retraktation in v. 23 unsäglich matt und abgeschmackt ist. Darum war ich geneigt, diesen Vers, obgleich er von Tertullian (de anima 50) bezeugt ist, für einen Zusatz zu halten und $\mu$ éveıv auf das Schlafen im Grabe zu deuten. Aber es war wohl ein Fehler, in dem Herrenspruch über den Lieblingsjünger eine Glorifikation des ephesischen Johannes zu sehen, die dem Martyrium des Petrus äquivalent sein sollte. Der Schreiber der Verse wollte nichts anderes als den Lieblingsjünger, den er 20, 24 für den Verfasser des Evangeliums ausgibt, so kennzeichnen, daß man auf den ephesischen Johannes, dessen Legende schon existierte, raten mußte: darum brachte er sein friedliches Ende in Gegensatz zum Martyrium des Petrus. Das mußte nun aber in die Unterredung des-Herrn mit Petrus, die der Interpolator schon vorfand, hineingebracht und in die Form einer Weissagung Jesu gepreßt werden. Ein Martyrium läßt sich leicht als Orakel formulieren, aber für die 
Weissagung eines ruhigen Abscheidens ist die prophetische Pointe schwerer zu finden; der Interpolator ist denn auch daran gescheitert und hat eine Tradition über den ephesischen Johannes erfunden, die er gleich darauf für ein Mißverständnis erklären muß. Es heißt seine Absichten gründlich verkennen, wenn man verlangt, daß er, um Johannes Petrus gleichzustellen, ja nur auf die Öberlieferung von dessen Martyrium hätte zurïckzugreifen brauchen; dem Manne kam es nicht darauf an, den wirklichen Zebedaeussohn Johannes zum Verfasser des Evangeliums zu machen, sondern den legendarischen Johannes, der der Schutzpatron von Ephesus war. Wenn Spitta nun gar auch hier ein Wort Jesu wittert, das ursprünglich ganz anders gemeint war und bedeutete: "Ivenn ich will, daß jener Jünger vorläufig noch in seinen alten Verhältnissen bleibt, während du mir als Hirt meiner Schafe folgst, was geht es dich an", so muß ich es ablehnen, dergleichen zu wider-

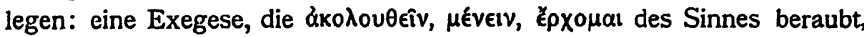
den sie in dem gegebenen Zusammenhang haben müssen und für jeden christlichen Leser hatten, entzieht sich der Diskussion.

Wie Evangelium, Apokalypse, der erste Brief dem Apostel Johannes und zwar dem der ephesischen Legende durch, leicht kenntliche Interpolationen zugeschrieben sind, so ist die Adresse zweier echter, lebensvoller Briefe, des zweiten und dritten, verstümmelt, um ihnen den gleichen Ursprung vindizieren zu können. Es ist nicht nötig, vielleicht nicht einmal ratsam, einem Manne das Verdienst zuzuschreiben, diese sehr verschiedenartigen Produkte der altchristlichen Literatur dadurch vor dem Untergang gerettet $z u$ haben, daß er sie mit einer Etikette versah, die sie für die Aufnahme in den Kanon legitimierte; hat ein solches Treiben einmal angefangen, so findet es leicht Fortsetzer, wie das Beispiel der apostolischen Konstitutionen, der apostolischen Kanones und der interpolierten ignatianischen Briefe zeigt, die man nicht auf einen Fälscher zurückführen kann. Eigentümlich ist, daß das Evangelium und die Apokalypse keine Originalwerke mehr waren, sondern mehrfache Überarbeitungen erlitten hatten, als sie den apostolischen Stempel erhielten; ferner daß der Verfasser des zweiten und dritten Briefes in irgend einem Zusammenhang mit dem des ersten und einem der Bearbeiter des Evangeliums steht: die letzten Herausgeber setzen eine literarische Bewegung fort, die schon im Gang war; sie stehen unter dem Druck des neuen, von der Rechtsfiktion der apostolischen Succession der Bischöfe entlehnten Gedankens, daß auch die literarische Überlieferung über das Evangelium direkt auf die Apostel zurückgeführt werden müsse. Da sie mit ziemlicher Sicherheit in der Provinz 
Asien zu suchen sind, so ist es nicht zu kühn, auch den Verfasser des zweiten und dritten Briefes, den ersten und die Bearbeitungen des Evangeliums und der Apokalypse dorthin zu verlegen. Insofern bekommt die aus der ephesischen Legende von Johannes abgeleitete Meinung, daß das vierte Evangelium in Kleinasien entstanden ist, Recht, da nicht die Grundschrift, sondern das überarbeitete Evangelium dasjenige ist, das die Entwicklung des Christentums in folgenschwerer Weise bestimmt hat: nur muß man jeden Gedanken an den geschichtlichen Johannes fahren lassen. Der bringt alles immer wieder in Verwirrung und hindert immer wieder, ein wichtiges Stück kleínasiatischer Kirchengeschichte aus der literarischen Analyse zu gewinnen. Der Chiliast Papias preist das Johannesevangelium als das wahre und apostolische; der Paraklet treibt durch ein Mißverständnis im Montanismus ein neues Urchristentum hervor; die Apokalypse ist immer eine gefährliche Waffe der Chiliasten gewesen und geblieben bis auf den heutigen Tag; der Verfasser der beiden kleinen Briefe eifert wider die offizielle Kirche, die an die Wiederkunft des Herrn im Fleisch nicht glaubt: das sind alles Erscheinungen, die in einen Zusammenhang gebracht werden müssen. Der werdende Montanismus ist es getvesen, der die johanneische Literatur nicht geschaffen, aber zusammengebracht und mit Benutzung der ephesischen Legende zu apostolischer Dignität erhoben hat. Nicht ohne Widerspruch zu finden; die älteste valentinianische Gnosis ignoriert das Johannesevangelium in ihrem Schriftbeweis und der orthodoxe römische Presbyter Gaius, aus dem Epiphanius unsinniger Weise die Sekte der Aloger herausgesponnen hat, bekämpfte mit vortrefflichen, nie widerlegten Gründen die Authentie der Apokalypse und des Evangeliums gegen den Montanisten Proklos; es ist charakteristisch, daß er gegen den Heroenkult der Kleinasiaten die Gräber der Apostel in Rom ausspielt. Diesmal behielten die Kleinasiaten Recht, nebenbei gesagt einer von den vielen Gegenbeweisen gegen die jetzige Mode, Rom für das Zentrum der altkirchlichen Entwicklung zu halten: nicht nur bei dem Kleinasiaten Irenaeus und dem Montanisten Tertullian, sondern auch bei dem Alexandriner Clemens ist jeder Zweifel an der Echtheit des Logosevangeliums geschwunden, und der Syrer Tatian sucht die Behauptung, es widerspreche den Synoptikern, durch die Tat zu widerlegen. Die Streitschrift des Gaius gegen Proklos mußte umgearbeitet und der Polemik gegen das Evangelium entkleidet werden, um als Waffe gegen die Phryger weiter gebraucht werden zu können.

Etwa um 150 muß das vierte Evangelium abgeschlossen und mit dem johanneischen Siegel versehen in die Welt hinausgegangen sein. Nichts 
stcht im Wege, anzunehmen, daß die Korrektur, die in den Apostelakten $(12,2)$ den Namen des Johannes entfernt hat, später erfolgt ist. Dagegen kann dies nicht der Grund dafür sein, daß das Orakel über die Zebedaeussöhne bei Lukas fehlt: das hat andere Gründe gehabt, die sich nicht mehr erraten lassen.

Es hatte nur so lange Sinn, darüber zu diskutieren, ob die Zebedaeussöhne durch ihr Martyrium den Anspruch auf die Ehrenplätze zur Seite des Herrn erworben hatten, als sie von den Zwölf die einzigen Märtyrer waren: die im Markusevangelium erhaltene Geschichte muß älter als das Todesjahr des Petrus, 64, sein. Da ferner die Apostelakten die Verfolgung, welche Jakobus, dem Bruder des Johannes, das Leben kostete und Petrus aus Jerusalem vertrieb, mit dem Tode des Agrippa verknüpfen, der sicher im Frühjahr 44 erfolgte, ist das Martyrium der Zebedaeussöhne mit größter Wahrscheinlichkeit in das Jahr 43/44 zu setzen: es ist das erste sichere Datum der Kirchengeschichte, von dem aus alle vorhergehenden Ereignisse bestimmt werden müssen. Zunächst und vor allem die Zusammenkunft des Paulus mit den "Säulen" in Jerusalem: sie muß vorher und zwar kurz vorher erfolgt sein und ist wahrscheinlich der Ainlaß der Verfolgung gewesen. Das stimmt allerdings zu der Erzählung der Apostelgeschichte nicht; aber es ist nachgewiesen, daß das Stück 15, $1-16,4$ auf dieselben Ereignisse geht, die 11,27-30 erzählt werden, und daß die Missionsreise des Paulus, über die die cc. 13 und 14 berichten, nicht vor, sondern nur hinter die $\mathrm{Zu}$ sammenkunft fallen kann. Öber diesen Punkt herrscht zwischen Spitta, Wellhausen und mir eine erfreuliche Übereinstimmung, so daß ich darüber nicht weiter $z \mathrm{u}$ reden brauche. Was Spitta nun hindert, uns zuzugeben, daß die 15, 1 ff. erzählte Reise des Paulus nach Jerusalem an die Stelle der $11,27 \mathrm{ff}$. berichteten $\mathrm{zu}$ schieben ist, bekenne ich nicht $\mathrm{zu}$ verstehen. Auch wenn man die Darstellung des Galaterbriefes zunächst beiseite hält und nur die Erzählung der Apostelakten betrachtet, ergeben sich die ärgsten Widersprüche und Ungereimtheiten. Eine planmäßig von der Regierung in Szene gesetzte Verfolgung, nicht etwa ein bloßer Pogrom ist über die Urgemeinde hereingebrochen, die Führer sind in erster Linie aufs Korn genommen, um auf die Weise die Organisation der Gemeinde $\mathrm{zu}$ sprengen. Und bald darauf versammeln sich die nSäulen" mit auswärtigen Missionaren, beratschlagen und beschließen mit.ihnen, als wenn von Verfolgung und Gefahr keine Rede wäre. Petrus war bei der Verfolgung entkommen $(12,17)$; bei jener Zusammenkunft ist er wieder in Jerusalem, ohne daß irgendwo von seiner Rückkehr die Rede ist: sie wäre ein kühnes Wagnis gewesen. Und noch 
dazu wird die Flucht in der sonderbarsten Weise ins Unbestimmte

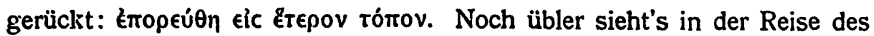
Barnabas und Paulus aus: sie kommen unmittelbar vor der Verfolgung in Jerusalem an, erleben sie dort als Zuschauer, denen nichts geschieht, und spazieren nach dem Tode Agrippas nach Antiochien zurück, wie Beamte, die eine gewöhnliche Dienstreise erledigt haben. Wer sich diese Inkongruenzen überlegt mit dem Hinblick auf den möglichen Hergang, nicht nur mit der Absicht, Quellen zu scheiden, der ist gegen den Spittaschen Einwand gefeit, daß „die großen Einschübe 12,1-24 und 15, 1-33 aus dem Zusammenhange derselben Schrift stammen“. Spitta übersieht, daß die Berichte, die in den Apostelakten zusammengearbeitet sind, sich nicht reinlich herauspräparieren lassen und daß ein Schluß wie der, den er hier zieht, ein methodischer Fehler ist, weil er die Tätigkeit des Redaktors nicht in Anschlag bringt. Weil dieser zwei Berichte sowohl von der Reise des Paulus zur Zusammenkunft in Jerusalem wie von der auf diese Zusammenkunft folgenden Missionsreise für Erzählungen verschiedener Ereignisse hielt und sie fälschlich vor und hinter die Verfolgung stellte, war er gezwungen, die entstehenden Ungereimtheiten einigermaßen durch redaktionelle Flicken zu verkleistern. Er verschweigt, wohin Petrus gegangen ist, weil er ihn nachher wieder in Jerusalem braucht, um inn mit Paulus zusammenzubringen, und doch von einer Rückreise des Petrus nach Jerusalem in seiner Vorlage nichts gefunden hat, und er läßt Paulus und Barnabas erst nach der Verfolgung von Jerusalem zurückkehren, weil diese Rückkehr zwar die richtige Einleitung zu ihrer Missionsreise ist, die Verfolgung aber von ihm zu früh eingelegt ist. Sie hätte hinter cc. 13 und 14 gehört, konnte aber unmöglich vor den zweiten Bericht von Paulus' und Barnabas' Reise nach Jerusalem und die sich daran anschließennde Zusammenkunft mit den Säulen gestellt werden. Da traten die Folgen der fehlerhaften Dublierung zu grell hervor, und weil der Redaktor den Sitz des Fehlers nicht erkannte, wußte er sich nicht anders zu helfen, als indem er die Verfolgung dahin stellte, wohin die Zusammenkunft des Paulus mit den Säulen gehört hätte.

Aus den Apostelakten allein würde der wahre Sachverhalt nicht mit Sicherheit erraten werden können; erst die Erzählung des Galaterbriefes macht es möglich, unter den Verschiebungen und Übermalungen des Redaktors die historisch brauchbaren Grundiagen seiner zusammengeklitterten Erzählung zu erkennen. Jene Erzählung lehrt, daß Paulus' Mission die Gegenden von Antiochien und Tarsos noch

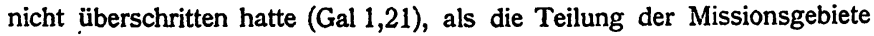


in Jerusalem stattfand: also stchen Act 13 und 14 an falscher Stelle, die kürzere, schattenhafte Wiederholung $15,35-16,4$ an richtiger. Aus ihr folgt ferner, daß Johannes noch am Leben war, als Paulus zu der Beratung nach Jerusalem kam: also ist der inhaltlose, schlechtere Bericht 11,29.30 chronologisch richtiger, weil er vor der Verfolgung steht, als der ausführliche und bessere, freilich durch Ubermalungen entstellte, der im c. 15 nachgetragen wird. Der Galaterbrief schafft auch die Aufklärung über die Flucht des Petrus, die durch den Redaktor von Act 12,17 vorenthalten ist. Petrus ist wegen der Verfolgung von Jerusalem nach Antiochien gegangen und höchstwahrscheinlich nie wieder zurückgekehrt.

Ist also die zweite Reise des bekehrten Paulus nach Jerusalem ins Jahr $43 / 44$ zu setzen, so fällt die erste 13 Jahre früher, 30/31, die Bekehrung zwei Jahre davor, 28/29. Ich habe, wie meine Ausführungen in der Darstellung des Paulus, die voriges Jahr erschienen ist (Charakterköpfe 2,110), jedem, der aufmerksam liest, verraten können, auch vor Spittas Polemik eingesehen, daß meine frühere Deutung von $\mathrm{Gal}$ 1, 18. 2,1 zu gewaltsam ist und ich mir die Schwierigkeit selbst geschaffen hatte, weil ich mich von dem traditionellen Glauben an das $\mathrm{Lk} 3,1$ überlieferte Datum nicht losmachen konnte. Wenn aber eine unbefangene Exegese der von Paulus angegebenen Intervalle mit Notwendigkeit $\mathrm{zu}$ der Alternative führt, entweder das Jahr 43/44 für Paulus' zweite Reise nach Jerusalem und die Verfolgung des Agrippa aufzugeben oder das lukanische Datum von $28 / 29$ für ungenau und willkürlich $z u$ halten, so kann eine methodische Kritik sich nur für die letztere Möglichkeit entscheiden. Es ist in ungewöhnlich pomphafter Form gegeben, und gerade das macht es verdächtig. Denn Lukas ist nicht imstande gewesen, die Paralleldaten alle richtig zu formulieren: zwei Hohenpriester gehören nicht in eine Zeitangabe, die auf Präzision Anspruch macht, und der Tetrarch Lysanias von Abilene ist längst als ein grober Fehler erkannt. ${ }^{1}$ Der Evangelist handhabt eine Form, die er aus einer fremden Sphäre übernommen hat und der er daher nicht gewachsen ist; sie stammt aus der profanen Historiographie, die die Gewohnheit hat, wichtige Ereignisse, sonderlich solche, mit denen die eigentliche Hauptdarstellung beginnt, durch umständliche Datierungen und Synchronismen hervorzuheben: Thukyd. 2, 1 und Polyb. 1,3 sind bekannte Beispiele. Lukas beging ferner, als sein schriftstellerischer Ehrgeiz ihn trieb, diese Form auf das Evangelium zu übertragen, den Fehler, daß er die Datierung

1 Nachr. d. Gött. Ges. d. Wiss. 1906, 370 f. 
an das hing, was zwar von jeher als der Anfang des Evangeliums von Jesus Christus galt, aber sich zeitlich in keiner Weise fixieren ließ, nämlich an die Prophezeiung des kommenden Messias durch Johannes den Täufer. Ganz abgesehen davon, daß die Christen die Messiaspredigt des Täufers mit Unrecht auf Jesus bezogen, war diese kein singuläres Ereignis, das durch eine Überlieferung an ein bestimmtes Jahr geknüpft war: sie war keine Prophetenrede, die wie die des AT in einem bestimmten Moment erging, sondern eine fortgesetzte Tätigkeit; wuchs doch eine ganze Sekte aus ihr hervor. Es bleibt also nichts anderes übrig als anzunehmen, daß Lukas die dem damaligen palaéstinischen Gebrauch entsprechende Angabe des Kaiserjahres von irgendeinem nicht mehr zu bestimmenden Anlaß auf die Täuferpredigt übertrug; ivenn die alte Kirche als diesen Anlaß Jesu Tod oder Jesu Taufe durch Johannes statuierte, so hat das für die historische Forschung nicht die mindeste Verbindlichkeit, und als die einzig brauchbare Datierung für das Leben Jesu bleibt nur die schon von Tacitus bezeugte, absolut unumstößliche übrig, daß er unter Pontius Pilatus gestorben ist. Daraus ist ein festes Jahr nicht zu gewinnen, auch durch die immer wieder vergeblich unternommenen Osterrechnungen nicht, die insgesamt das wenige ignorieren, was über die damalige Art der Juden, die Zeit des Pascha festzusetzen, gewußt werden kann, und den Freitag der Passion für sicher überliefert halten. ${ }^{1}$

Übrigens will ich nicht behauptet haben, daß Jesus mehr als ziwei oder höchstens drei Jahre vor dem Pascha von 29 gestorben ist; der Ansatz von Paulus' Bekehrung auf 28/29 läßt sich auch mit einer geringen Zurückschiebung durchaus vereinen. Ich habe mich leider auch noch in meiner Darstellung des Paulus durch Mommsens Autorität dazu verführen lassen $z u$ leugnen, daß Paulus vor der ersten Reise nach der Bekehrung (Gal 1,18) in Jerusalem gewesen sein könne, so daß er das werdende Christentum nicht dort, sondern in Damaskus kennen gelernt haben muß. Das darf aus Gal 1,22 nicht gefolgert werden:

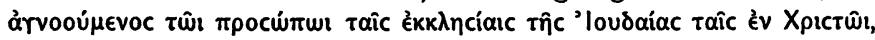

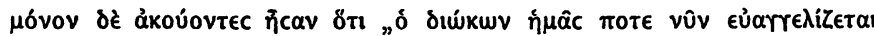

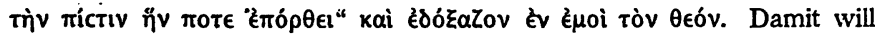
der Apostel nur sagen, daß er nach seiner Bekehrung mit den christlichen Gemeinden in und um Jerusalem keine nähere Beziehung unterhalten hatte, nicht in ihre Gottes̀dienste gegangen und mit keinem der Brüder persönlich bekannt geworden war. Darauf allein kommt es ihm

1 Abhdlg. d. Gött. G. d. Wiss. N. F. VIIl 6, 144. Diese Zeitschr. 7, 8. 31 f. 
im Zusammenhang an; ob er vor der Bekehrung in der heiligen Stadt gewesen war oder nicht, war für den Beweis, den er führen will, gleichgültig. $\mathrm{Daß}$ er es wirklich gewesen ist, folgt nicht aus den entstellten Erzählungen der Apostclakten, wohl aber aus Paulus' eigenen

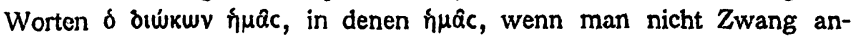
wenden will, nur auf die Gemeinden in Judäa bezogen werden kann. Mochten auch einzelne wissen, wie der Verfolger aussah, so war er darum doch den Gemeinden noch nicht von Angesicht bekannt, das wurde er nur, wenn er sich ihnen als Gläubiger und Bruder vorstellte. Es wird also doch richtig sein, daß Paulus in Jerusalem die Gemeinde des Auferstandenen kennen lernte und den Geist Jesu spürte, zunächst als Gegner, dann so stark, daß die Offenbarung von Damaskus einschlagen konnte. Viel Zeit brauchte von Jesu Tod bis dahin nicht verstrichen zu sein: Gottes Ernten reifen schnell.

Ostern 1910. 